\title{
Studies on the Effect of Organic Amendments and Bio-agents on Seedling Growth of Acid Lime (Citrus aurantifolia Swingle) cv. Balaji
}

\author{
S.M. Rajesh Naik*, K.T. Venkataramana, L. Mukundalakshmi, \\ R. Nagaraju and G. Srinivas Rao \\ Department of Fruit Science, College of Horticulture, Dr. YSRHU, Anantharajupeta- 516105, \\ Andhra Pradesh, India \\ *Corresponding author
}

\begin{tabular}{|l|}
\hline Ke y w or d s \\
Acid lime, \\
Arbuscular \\
mycorrhiza fungi, \\
Azotobacter
\end{tabular}

A B S T R A C T

The experiment was carried out at AICRP on Fruits, Citrus Research Station, Tirupati, during 2016-2017 to study the effect of organic amendments and bio-agents on growth of differently aged seedlings of acid lime (Citrus aurantifolia Swingle) cv. Balaji. The experiment was conducted in completely randomized design with three replications. The treatments consisted of 21 treatments with different potting media, organic amendments and bio-agents and their effect was studied on three age group acid lime seedlings. In two months old acid lime seedlings experiment the results indicated that maximum plant height, number of leaves, leaf area, stem girth and Chlorophyll content per seedlings at 150 days after transplanting were recorded in the potting media containing soil, sand, cocopeat @ 1:1:1 v/v, neem cake $(20 \mathrm{~g})$ and $A M$ @ $5 \mathrm{~g} / \mathrm{bag}$ each per bag. Whereas, highest stem girth was observed in the potting media containing soil, sand, vermicompost@1:1:1 v/v, neem cake (20g) and $A M$ @ $5 \mathrm{~g} / \mathrm{bag}$ each per bag. In case of three and four months old acid lime seedlings maximum plant height, number of leaves, stem girth, leaf area and Chlorophyll content per seedlings were recorded in the potting media containing soil + sand + vermicompost + Arbuscular mycorrhiza $(5 \mathrm{~g})+$ neem cake $20 \mathrm{~g}$ each per bag. However, minimum growth parameters were recorded when soil, sand, FYM @ 1:1:1 v/v was used as potting media in case of 2, 3, 4 months old seedlings.

\section{Introduction}

In India, among the fruit crops citrus species covers an area of $10.4 \%$ of major fruit crops with an area $268.4 \mathrm{Mha}$, with the production of 2950.4 MT and the productivity of 11.0 MT/ha (Anonymous 2015-16). The major lime producing states are Andhra Pradesh, Maharashtra, Gujarat, Karnataka, Tamil Nadu, Assam and Chhattisgarh. Andhra Pradesh stands first in the country both in area (38850 hectares) and production (582743 MT) for acid lime. Major acid lime growing areas in the state are Nellore, Kadapa, Ananthapuramu, Kurnool, Guntur, East and West Godavari districts. The annual yield varies from 3000 to 5000 fruit per tree.

Supply of quality planting material is an important prerequisite for realizing potential 
yields by the farmers. Acid lime is a slow growing plant and is commercially propagated through seeds. Because of its slow growing nature it takes nearly 12 months to attain saleable girth and height of plants in the poly bags. As there is heavy demand for planting material from farmers, it is necessary to bridge the gap between demand and production. Hence there is need to speed up the growth of seedlings grown in poly bags by making required changes in potting media composition there by reducing the time taken to reach saleable growth.

A potting medium is a composition of organic materials formulated to achieve desirable chemical and physical needs required by the crop to attain its potential growth and development. Good container-media management is basic to the production of quality container-grown citrus nursery plants. Farmyard manure has been the main basic source of organic matter for the supply of essential minerals needed by the plants. Azotobacter is not only associated with the process of nitrogen fixation and improving nitrogen of plants, but also with the supply of biologically active compounds such as vitamins and gibberellins. The mutualistic association of Arbuscular mycorrhiza (AM) with roots of most terrestrial plants is well known which could enhance plant growth and alleviate salt stress. The mycorrhizal fungi Increase surface area for nutrient absorption and transport them back to the plant. The nutrients $\mathrm{P}, \mathrm{Zn}, \mathrm{C}, \mathrm{N}, \mathrm{Cu}$ and $\mathrm{S}$ absorbed and translocated to the host and produces hormones like auxin, cytokinins, gibberellins and vitamins. AM fungi acts as accessories to the root hairs in the process of nutrient absorption and mobilization, there by facilitate nutrient translocation from the soil and root cortical parenchyma to xylem elements of the host plant.

In view of the potential roles played by the organic manures and bio agents in augmenting plant growth, an attempt is made to utilize these materials in the potting media in different doses and combinations with the objective of boosting the seedling growth of acid lime variety $\mathrm{Cv}$. Balaji.

\section{Materials and Methods}

The experiment was conducted at Citrus Research Station, Department of Horticulture, Dr. Y.S.R. Horticultural University, Tirupathi, Andhra Pradesh, during the year 2016-17. The experiment was laid out in a Completely Randomized Design (CRD) with three replications and 21 treatments viz., $\mathrm{T}_{1}: \mathrm{FYM}$ +Sand +soil (control) @ 1:1:1 v/v, T2: Vermicompost +Sand + Soil@ 1:1:1 v/v, $\mathrm{T}_{3}:$ Cocopeat + Sand + Soil@ 1:1:1 v/v, $\mathrm{T}_{4}: \mathrm{T}_{1}$ + Neem cake, $\mathrm{T}_{5}: \mathrm{T}_{1}+$ Neem cake + Azotobacter, $\mathrm{T}_{6}: \mathrm{T}_{1}+$ Neem cake + Arbuscular mycorrhiza, $\mathrm{T}_{7}: \mathrm{T}_{1}+$ Castor cake, $\mathrm{T}_{8}: \mathrm{T}_{1}+$ Castor cake + Azotobacter, $\mathrm{T}_{9}: \mathrm{T}_{1}+$ Castor cake + Arbuscular mycorrhiza, $\mathrm{T}_{10}: \mathrm{T}_{2}+$ Neem cake, $\mathrm{T}_{11}: \mathrm{T}_{2}+$ Neem cake + Azotobacter, $\mathrm{T}_{12}$ : $\mathrm{T}_{2}+$ Neem cake + Arbuscular mycorrhiza, $\mathrm{T}_{13}: T_{2}+$ Castor cake, $T_{14}: T_{2}+$ Castor cake + Azotobacter, $T_{15}: T_{2}+$ Castor cake + Arbuscular mycorrhiza, $T_{16}: T_{3}+$ Neem cake, $T_{17}: T_{3}+$ Neem cake + Azotobacter, $T_{18}: T_{3}+$ Neem cake + Arbuscular mycorrhiza, $T_{19}: T_{3}+$ Castor cake, $T_{20}: T_{3}+$ Castor cake + Azotobacter, $T_{21}: T_{3}+$ Castor cake + Arbuscular mycorrhiza. Hundred seedlings were maintained per treatment and each replication.

The acid lime cultivar used in this study was Balaji, released from CRS, Tirupati, which is under commercial cultivation in Andhra Pradesh. Fully matured acid lime fruits were harvested manually from trees and seeds were carefully extracted manually by cutting the fruits and squeezing out the seeds. Extracted seeds were thoroughly washed with clean water and later they were spread on the clean floor in thin layer under the shade and dried for 2 to 3 days. Thoroughly dried seeds were 
used for sowing on the raised nursery beds. Before sowing the seeds were treated with Diathane M-45@3g per kg seed and shade dried for 2 hours. Black polyethylene bags of gauge 100 microns and having dimensions of $6 \times 8$ inches were used for filling of potting media. Poly bags were filled with potting media consisted of soil, sand, vermicompost, cocopeat, farm yard manure in different proportions $(1: 1: 1 \mathrm{v} / \mathrm{v})$. Healthy seedlings with vigorous growth having uniform height were transplanted at 2 months age, 3 months age and 4 months age separately in to the poly bags containing media in different combinations. Before transplanting to bags the seedling roots were washed under the running water and the roots dipped in fungicidal solution (Diathane M-45 @ 3g per liter of water) for 2 minutes as a preventive step against soil borne pathogens.

In each treatment ten plants were randomly selected from each replication for recording growth parameters at monthly interval starting from 60 days after transplanting to 150 days after transplanting. Observation on seedling height, stem diameter, number of leaves, SPAD, leaf area was recorded. The seedling height was measured from collar region (ground level) to tip of seedlings using metric scale, Number of leaves was recorded by counting total functional leaves per seedling, stem girth was measured with "Vernier calipers" at $1 \mathrm{~cm}$ height from the base of stem, third leaf from the top of each seedling was used for recording SPAD Chlorophyll Meter readings and leaf area was calculated using leaf area meter (Li-COR model LI 3000) and 10 leaves were taken for plant in each replication for recording leaf area and the average was calculated.

\section{Results and Discussion}

The significantly highest plant height (26.83 $\mathrm{cm}$ ) was recorded with $\mathrm{T}_{18}$ [Cocopeat + Sand + Soil + Neem cake (20g) + Arbuscular mycorrhiza $(5 \mathrm{~g})$ ] in two months old seedlings (Table 1). The treatment closely followed by $\mathrm{T}_{12}(24.26 \mathrm{~cm})$ [Vermicompost + Sand + Soil + Neem cake + Arbuscular mycorrhiza (5g)]. Respectively least plant $(18.16 \mathrm{~cm})$ height were recorded with $\mathrm{T}_{1}(\mathrm{FYM}+$ Sand + Soil $)$ at $60,90,120$ and 150 days after transplanting. Similar results have been recorded with seedlings transplanting at three months age where in $\mathrm{T}_{18}$ gave significantly tallest seedlings $(37.81 \mathrm{~cm})$ closely followed by $\mathrm{T}_{12}$ (36.64), however with regards to the seedlings of four months old, significantly highest plant height $(39.33 \mathrm{~cm})$ have been recorded with $\mathrm{T}_{12}$ closely followed by $\mathrm{T}_{18}$ (38.76). In case of 2 months, 3 months and 4 months old seedlings least plant height were observed in $T_{1}$. Increase in plant height in treatment -18 and 12 might be due to the fact that these organic amendments i.e. vermicompost, cocopeat, neem cake besides increasing water holding capacity, these are also rich in nutrients. Amendments release nutrient slowly for longer periods, hence the availability of nutrients is continuous, thus contributed to vigorous growth of the seedlings. Similar findings were also reported by Bhardwaj (2013) in papaya, Patil et al., (2013) in Rangpur lime seedlings, Kamble et al., (2010) in mango seedlings (Fig. 1).

In case of two months old seedlings highest number of leaves were observed in $\mathrm{T}_{18}$ (29.64) followed by $\mathrm{T}_{12}$ (28.68) and lowest numbers of leaves were recorded with $\mathrm{T}_{1}(\mathrm{FYM}+$ Sand +soil) 18.90 at 150 days after transplanting (Table 2). Similar results have also been recorded with the seedlings of three months old seedling maximum number of leaves was recorded with $\mathrm{T}_{12}$ (32.18) and in four months old seedlings maximum number of leaves was observed In $\mathrm{T}_{12}$ (33.18) at 150 days in case of 3 and 4 months old seedlings lowest number of leaves was observed in $\mathrm{T}_{1}$. Combination of organic amendments i.e., cocopeat and vermicompost in association of bio agent $A M$ appears to have played a vital role in 
increasing overall growth of seedlings leading close conformity with those of Rakesh et al., to the production of more number of leaves in (2012) in acid lime seedlings and Sharma treatments 18 and 12. These findings are in (2009) in citrus seedlings.

Fig.1 Effect of organic amendments and bio agents on plant height of 2, 3 and 4 months old acid lime seedings $\mathrm{Cv}$. Balaji
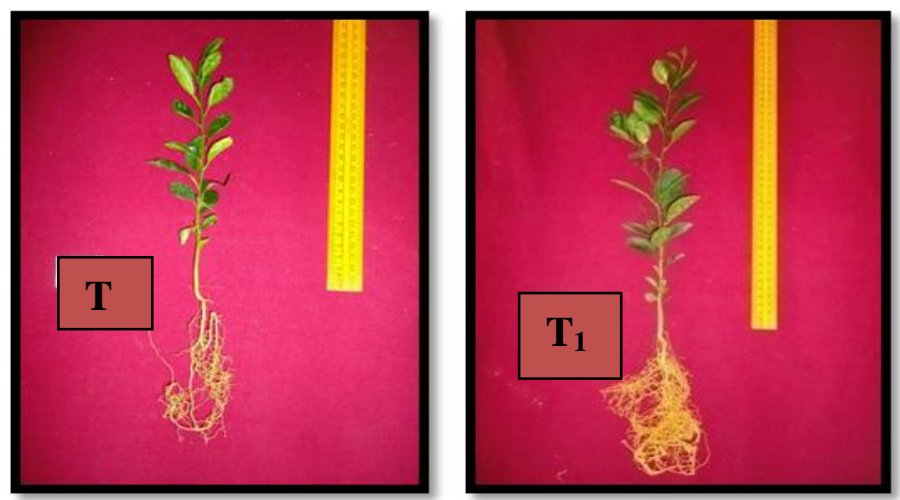

2 months old seedlings

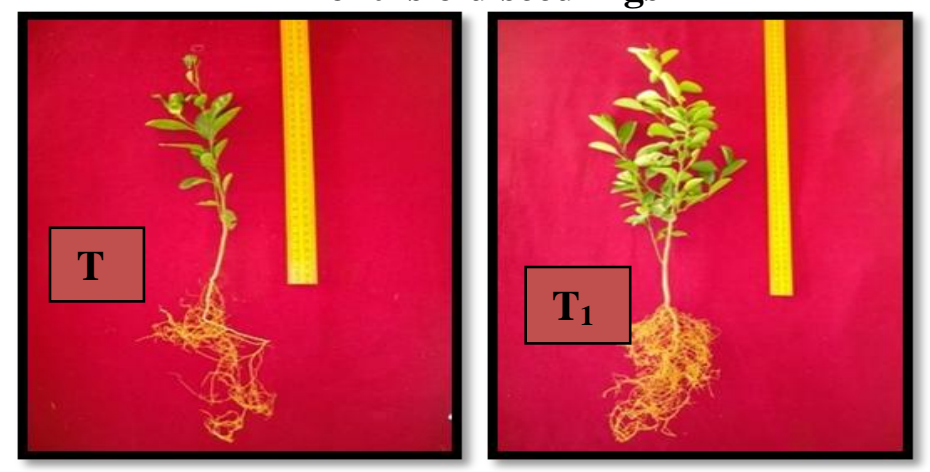

3 months old seedlings
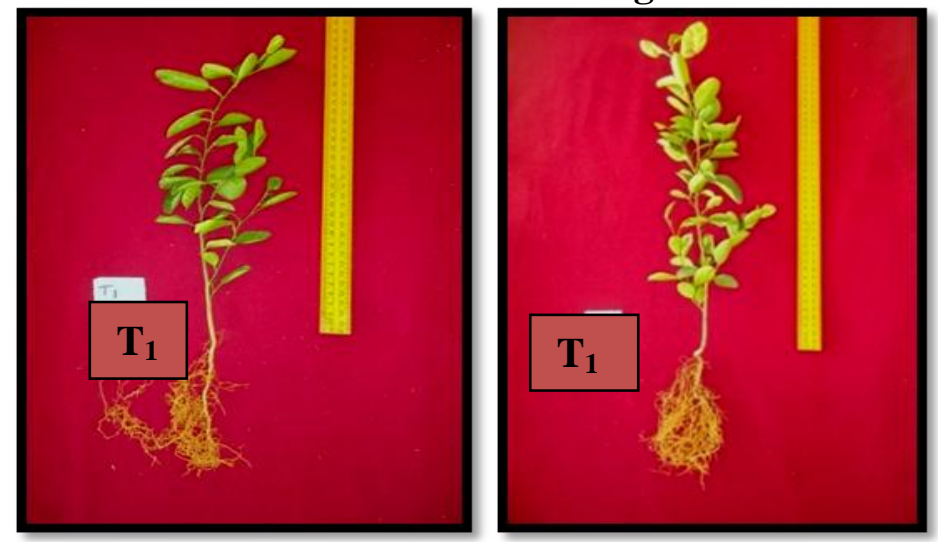
Table.1 Effect of organic amendments and bio-agents on plant height (cm) of 2, 3 and 4 months old acid lime seedlings cv. Balaji

\begin{tabular}{|c|c|c|c|c|c|c|c|c|c|c|c|c|}
\hline \multirow{2}{*}{ Treatment } & \multicolumn{4}{|c|}{2 months old } & \multicolumn{4}{|c|}{3 months old } & \multicolumn{4}{|c|}{4 months old } \\
\hline & DAT 60 & $\begin{array}{c}\text { DAT } \\
90\end{array}$ & DAT 120 & $\begin{array}{r}\text { DAT } \\
150\end{array}$ & DAT 60 & $\begin{array}{c}\text { DAT } \\
90\end{array}$ & $\begin{array}{r}\text { DAT } \\
120\end{array}$ & $\begin{array}{c}\text { DAT } \\
150\end{array}$ & DAT 60 & DAT 90 & $\begin{array}{c}\text { DAT } \\
120\end{array}$ & $\begin{array}{c}\text { DAT } \\
150\end{array}$ \\
\hline FYM +Sand +soil (control) & 5.09 & 7.04 & 12.39 & 18.16 & 11.07 & 16.00 & 21.18 & 26.98 & 15.33 & 18.66 & 24.5 & 30.48 \\
\hline Vermicompost + Sand + Soil & 5.54 & 8.82 & 13.75 & 18.88 & 12.11 & 16.86 & 22.01 & 29.27 & 16.53 & 19.13 & 25.3 & 31.21 \\
\hline Cocopeat + Sand + Soil & 5.83 & 9.02 & 13.10 & 19.31 & 11.12 & 16.23 & 21.43 & 27.25 & 16.48 & 18.70 & 24.9 & 30.98 \\
\hline $\mathrm{T}_{1}+$ Neem cake & 6.06 & 9.54 & 14.49 & 20.38 & 11.25 & 16.90 & 21.98 & 28.32 & 16.90 & 21.18 & 26.1 & 31.90 \\
\hline $\mathrm{T}_{1}+$ Neem cake + Azotobacter & 6.42 & 9.87 & 15.18 & 20.92 & 14.27 & 19.87 & 25.18 & 30.40 & 17.13 & 22.72 & 29.1 & 34.15 \\
\hline $\mathrm{T}_{1}+$ Neem cake + Arbuscular mycorrhiza & 8.22 & 11.75 & 15.94 & 22.32 & 15.79 & 23.03 & 23.82 & 31.87 & 19.67 & 23.27 & 31.5 & 38.49 \\
\hline $\mathrm{T}_{1}+$ Castor cake & 5.96 & 8.98 & 14.06 & 19.17 & 13.41 & 17.26 & 21.24 & 28.15 & 17.52 & 20.49 & 25.1 & 32.09 \\
\hline $\mathrm{T}_{1}+$ Castor cake + Azotobacter & 6.50 & 10.88 & 14.77 & 19.96 & 14.57 & 19.61 & 22.27 & 28.89 & 17.07 & 19.14 & 26.5 & 32.75 \\
\hline $\begin{array}{l}\mathrm{T}_{9} \quad \mathrm{~T}_{1}+\text { Castor cake }+ \text { Arbuscular } \\
\text { mycorrhiza }\end{array}$ & 7.08 & 11.18 & 15.92 & 21.36 & 14.13 & 20.51 & 25.12 & 30.12 & 17.78 & 21.05 & 27.3 & 33.54 \\
\hline $\mathrm{T}_{10} \quad \mathrm{~T}_{2}+$ Neem cake & 6.35 & 9.88 & 13.86 & 19.48 & 13.08 & 18.35 & 22.36 & 28.69 & 16.70 & 19.58 & 25.3 & 31.70 \\
\hline $\mathrm{T}_{11} \mathrm{~T}_{2}+$ Neem cake + Azotobacter & 6.81 & 11.44 & 16.19 & 21.79 & 17.75 & 22.26 & 27.24 & 35.04 & 19.28 & 23.42 & 29.3 & 35.13 \\
\hline $\mathrm{T}_{12} \mathrm{~T}_{2}+$ Neem cake + Arbuscular mycorrhiza & 9.23 & 12.87 & 17.47 & 24.26 & 17.37 & 22.81 & 29.34 & 36.64 & 20.98 & 25.40 & 31.8 & 39.33 \\
\hline $\mathrm{T}_{13} \quad \mathrm{~T}_{2}+$ Castor cake & 6.45 & 11.76 & 15.41 & 19.55 & 15.19 & 19.04 & 23.06 & 29.24 & 17.71 & 21.10 & 25.9 & 31.43 \\
\hline $\mathrm{T}_{14} \mathrm{~T}_{2}+$ Castor cake + Azotobacter & 6.95 & 11.39 & 16.01 & 20.47 & 16.28 & 21.13 & 25.47 & 31.35 & 18.78 & 21.79 & 26.9 & 32.81 \\
\hline $\mathrm{T}_{15} \mathrm{~T}_{2}+$ Castor cake + Arbuscular mycorrhiza & 7.23 & 12.46 & 17.11 & 23.42 & 17.02 & 22.18 & 26.53 & 34.68 & 19.48 & 24.31 & 29.4 & 35.32 \\
\hline $\mathrm{T}_{16} \mathrm{~T}_{3}+$ Neem cake & 5.92 & 9.21 & 13.92 & 20.09 & 13.58 & 19.38 & 23.15 & 29.55 & 18.87 & 22.62 & 26.9 & 32.23 \\
\hline $\mathrm{T}_{17} \mathrm{~T}_{3}+$ Neem cake + Azotobacter & 7.36 & 12.16 & 16.10 & 22.40 & 16.68 & 21.08 & 25.90 & 34.23 & 19.15 & 24.59 & 29.7 & 34.74 \\
\hline $\mathrm{T}_{18} \quad \mathrm{~T}_{3}+$ Neem cake + Arbuscular mycorrhiza & 10.14 & 13.53 & 19.06 & 26.83 & 18.09 & 24.60 & 31.41 & 37.81 & 20.83 & 24.68 & 31.6 & 38.76 \\
\hline $\mathrm{T}_{19} \quad \mathrm{~T}_{3}+$ Castor cake & 6.57 & 11.27 & 15.88 & 21.54 & 15.10 & 19.75 & 24.09 & 30.46 & 18.05 & 21.83 & 26.8 & 32.46 \\
\hline $\mathrm{T}_{20} \quad \mathrm{~T}_{3}+$ Castor cake + Azotobacter & 7.02 & 11.80 & 16.00 & 22.52 & 14.66 & 18.37 & 24.19 & 31.12 & 17.32 & 22.34 & 28.0 & 33.19 \\
\hline $\mathrm{T}_{21} \mathrm{~T}_{3}+$ Castor cake + Arbuscular mycorrhiza & 7.23 & 12.46 & 16.94 & 24.18 & 15.60 & 20.52 & 26.95 & 33.31 & 19.04 & 23.42 & 30.3 & 36.20 \\
\hline Mean & 6.86 & 10.82 & 15.41 & 21.28 & 14.67 & 19.80 & 24.47 & 31.11 & 18.12 & 21.88 & 27.7 & 33.76 \\
\hline $\mathrm{SE}(\mathrm{m}) \pm$ & 0.30 & 0.37 & 0.30 & 0.56 & 0.60 & 0.38 & 0.57 & 0.5 & 0.33 & 0.50 & 1.24 & 1.14 \\
\hline C.D. $(5 \%)$ & 0.87 & 1.06 & 0.88 & 1.61 & 1.22 & 1.11 & 1.64 & 1.4 & 0.95 & 1.43 & 3.57 & 3.26 \\
\hline
\end{tabular}


Table.2 Effect of organic amendments and bio-agents on number of leaves of 2, 3 and 4 months old acid lime seedlings cv. Balaji

\begin{tabular}{|c|c|c|c|c|c|c|c|c|c|c|c|c|}
\hline \multirow{2}{*}{ Treatment } & \multicolumn{4}{|c|}{2 months old } & \multicolumn{4}{|c|}{3 months old } & \multicolumn{4}{|c|}{4 months old } \\
\hline & DAT 60 & DAT 90 & DAT 120 & DAT 150 & DAT 60 & DAT 90 & DAT 120 & DAT 150 & DAT 60 & DAT 90 & DAT 120 & DAT 150 \\
\hline $\mathrm{T}_{1} \quad \mathrm{FYM}+$ Sand +soil (control) & 5.88 & 10.74 & 14.83 & 18.90 & 8.90 & 13.85 & 18.59 & 22.18 & 10.24 & 16.41 & 20.92 & 23.18 \\
\hline $\mathrm{T}_{3} \quad$ Cocopeat + Sand + Soil & 7.12 & 12.08 & 18.31 & 21.49 & 9.28 & 14.48 & 18.74 & 22.75 & 12.25 & 17.18 & 22.52 & 25.36 \\
\hline $\mathrm{T}_{4} \quad \mathrm{~T}_{1}+$ Neem cake & 7.80 & 12.07 & 18.34 & 21.21 & 10.34 & 15.97 & 21.16 & 24.38 & 11.79 & 17.63 & 23.26 & 26.84 \\
\hline $\mathrm{T}_{5} \quad \mathrm{~T}_{1}+$ Neem cake + Azotobacter & 9.13 & 14.27 & 20.43 & 25.26 & 12.85 & 17.53 & 23.03 & 27.48 & 14.28 & 20.72 & 25.35 & 31.25 \\
\hline $\begin{array}{l}\mathrm{T}_{6} \quad \mathrm{~T}_{1}+\text { Neem cake }+ \text { Arbuscular } \\
\text { mycorrhiza }\end{array}$ & 9.65 & 15.17 & 21.66 & 26.31 & 13.37 & 18.85 & 22.81 & 29.32 & 14.59 & 21.41 & 27.30 & 32.29 \\
\hline $\mathrm{T}_{7} \quad \mathrm{~T}_{1}+$ Castor cake & 7.20 & 12.38 & 16.71 & 22.27 & 9.74 & 14.64 & 18.82 & 23.17 & 13.23 & 15.42 & 18.73 & 25.26 \\
\hline $\mathrm{T}_{8} \quad \mathrm{~T}_{1}+$ Castor cake + Azotobacter & 7.93 & 13.80 & 18.36 & 24.76 & 10.97 & 15.72 & 19.63 & 24.40 & 12.36 & 16.61 & 21.59 & 26.29 \\
\hline $\begin{array}{l}\mathrm{T}_{9} \quad \mathrm{~T}_{1}+\text { Castor cake }+ \text { Arbuscular } \\
\text { mycorrhiza }\end{array}$ & 8.56 & 13.37 & 19.73 & 25.49 & 12.38 & 16.68 & 20.35 & 26.81 & 14.14 & 19.29 & 25.20 & 30.12 \\
\hline $\begin{array}{ll}\mathrm{T}_{10} & \mathrm{~T}_{2}+\text { Neem cake }\end{array}$ & 7.34 & 13.91 & 19.20 & 23.21 & 10.46 & 14.59 & 18.87 & 24.29 & 11.46 & 16.81 & 21.31 & 24.18 \\
\hline $\mathrm{T}_{11} \quad \mathrm{~T}_{2}+$ Neem cake + Azotobacter & 8.17 & 15.73 & 21.61 & 25.95 & 13.31 & 16.81 & 21.15 & 25.27 & 14.61 & 17.92 & 22.25 & 27.60 \\
\hline $\mathrm{T}_{13} \mathrm{~T}_{2}+$ Castor cake & 7.87 & 12.81 & 19.37 & 23.80 & 11.60 & 15.95 & 19.26 & 23.98 & 12.49 & 17.29 & 20.14 & 25.50 \\
\hline $\mathrm{T}_{14} \mathrm{~T}_{2}+$ Castor cake + Azotobacter & 8.50 & 13.62 & 20.15 & 24.87 & 11.39 & 17.20 & 22.38 & 26.30 & 12.50 & 18.20 & 23.60 & 27.51 \\
\hline $\begin{array}{l}\mathrm{T}_{15} \quad \mathrm{~T}_{2}+\text { Castor cake }+ \text { Arbuscular } \\
\text { mycorrhiza }\end{array}$ & 8.87 & 14.12 & 21.03 & 26.10 & 12.62 & 18.13 & 22.48 & 28.71 & 13.84 & 19.12 & 26.87 & 31.74 \\
\hline $\mathrm{T}_{16} \quad \mathrm{~T}_{3}+$ Neem cake & 7.82 & 12.87 & 17.98 & 23.53 & 11.43 & 16.70 & 20.26 & 24.40 & 12.64 & 17.51 & 21.15 & 24.95 \\
\hline $\mathrm{T}_{17} \quad \mathrm{~T}_{3}+$ Neem cake + Azotobacter & 8.61 & 13.98 & 20.50 & 26.04 & 10.81 & 16.29 & 21.48 & 25.32 & 12.81 & 17.51 & 23.26 & 26.32 \\
\hline $\begin{array}{l}\mathrm{T}_{18} \quad \mathrm{~T}_{3}+\text { Neem cake }+ \text { Arbuscular } \\
\text { mycorrhiza }\end{array}$ & 11.37 & 16.65 & 23.30 & 29.64 & 13.92 & 20.76 & 24.49 & 30.75 & 15.59 & 21.31 & 27.82 & 32.73 \\
\hline $\mathrm{T}_{19} \quad \mathrm{~T}_{3}+$ Castor cake & 7.66 & 11.87 & 16.88 & 21.20 & 11.73 & 15.81 & 20.77 & 24.29 & 12.84 & 17.92 & 22.99 & 26.85 \\
\hline $\mathrm{T}_{20} \quad \mathrm{~T}_{3}+$ Castor cake + Azotobacter & 7.99 & 12.75 & 18.63 & 23.02 & 12.11 & 16.63 & 22.12 & 25.37 & 13.61 & 18.41 & 23.68 & 26.55 \\
\hline $\begin{array}{l}\mathrm{T}_{21} \quad \mathrm{~T}_{3}+\text { Castor cake }+ \text { Arbuscular } \\
\text { mycorrhiza }\end{array}$ & 8.83 & 14.20 & 19.93 & 25.71 & 13.28 & 18.71 & 22.73 & 28.07 & 13.89 & 19.16 & 25.84 & 29.18 \\
\hline Mean & 8.24 & 13.62 & 19.39 & 24.18 & 11.67 & 16.72 & 21.20 & 25.86 & 13.16 & 18.36 & 23.57 & 27.80 \\
\hline $\mathrm{SE}(\mathrm{m}) \pm$ & 0.32 & 0.43 & 0.50 & 0.41 & 0.52 & 0.72 & 0.83 & 0.90 & 0.52 & 0.58 & 0.98 & 1.19 \\
\hline
\end{tabular}


Table.3 Effect of organic amendments and bio-agents on stem girth (mm) of 2, 3 and 4 months old acid lime seedlings cv. Balaji

\begin{tabular}{|c|c|c|c|c|c|c|c|c|c|c|c|c|}
\hline \multirow{2}{*}{ Treatment } & \multicolumn{4}{|c|}{2 months old } & \multicolumn{4}{|c|}{3 months old } & \multicolumn{4}{|c|}{4 months old } \\
\hline & DAT 60 & DAT 90 & DAT 120 & DAT 150 & DAT 60 & DAT 90 & DAT 120 & DAT 150 & DAT 60 & DAT 90 & DAT 120 & DAT 150 \\
\hline $\mathrm{T}_{1} \quad \mathrm{FYM}+$ Sand +soil (control) & 0.87 & 1.08 & 1.21 & 1.35 & 1.18 & 1.34 & 1.63 & 1.85 & 1.74 & 1.85 & 1.97 & 2.08 \\
\hline $\mathrm{T}_{2} \quad$ Vermicompost + Sand + Soil & 0.95 & 1.12 & 1.33 & 1.52 & 1.20 & 1.35 & 1.66 & 1.87 & 1.51 & 1.85 & 1.96 & 2.13 \\
\hline $\mathrm{T}_{3} \quad$ Cocopeat + Sand + Soil & 0.89 & 1.15 & 1.31 & 1.47 & 1.22 & 1.40 & 1.67 & 1.91 & 1.41 & 1.86 & 1.95 & 2.11 \\
\hline $\mathrm{T}_{4} \quad \mathrm{~T}_{1}+$ Neem cake & 1.02 & 1.22 & 1.42 & 1.58 & 1.28 & 1.42 & 1.80 & 2.06 & 2.12 & 2.25 & 2.37 & 2.56 \\
\hline $\mathrm{T}_{5} \quad \mathrm{~T}_{1}+$ Neem cake + Azotobacter & 1.20 & 1.36 & 1.53 & 1.72 & 1.37 & 1.54 & 1.80 & 2.19 & 2.25 & 2.38 & 2.49 & 2.67 \\
\hline $\mathrm{T}_{6} \quad \mathrm{~T}_{1}+$ Neem cake + Arbuscular mycorrhiza & 1.35 & 1.54 & 1.70 & 1.95 & 1.51 & 1.76 & 1.96 & 2.42 & 2.40 & 2.49 & 2.66 & 2.83 \\
\hline $\mathrm{T}_{7} \quad \mathrm{~T}_{1}+$ Castor cake & 0.98 & 1.17 & 1.38 & 1.59 & 1.31 & 1.44 & 1.80 & 2.07 & 2.03 & 2.09 & 2.25 & 2.41 \\
\hline $\mathrm{T}_{8} \quad \mathrm{~T}_{1}+$ Castor cake + Azotobacter & 1.03 & 1.24 & 1.46 & 1.66 & 1.31 & 1.48 & 1.90 & 2.21 & 2.11 & 2.24 & 2.37 & 2.58 \\
\hline $\mathrm{T}_{9} \quad \mathrm{~T}_{1}+$ Castor cake + Arbuscular mycorrhiza & 1.12 & 1.28 & 1.49 & 1.70 & 1.39 & 1.58 & 1.79 & 2.16 & 2.25 & 2.31 & 2.47 & 2.64 \\
\hline $\mathrm{T}_{10} \quad \mathrm{~T}_{2}+$ Neem cake & 0.95 & 1.14 & 1.31 & 1.62 & 1.33 & 1.57 & 1.83 & 2.13 & 2.01 & 2.24 & 2.44 & 2.66 \\
\hline $\mathrm{T}_{11} \quad \mathrm{~T}_{2}+$ Neem cake + Azotobacter & 1.18 & 1.39 & 1.56 & 1.91 & 1.71 & 1.93 & 2.18 & 2.60 & 2.08 & 2.19 & 2.34 & 2.53 \\
\hline $\mathrm{T}_{12} \quad \mathrm{~T}_{2}+$ Neem cake + Arbuscular mycorrhiza & 1.58 & 1.73 & 1.90 & 2.56 & 1.81 & 2.05 & 2.43 & 3.13 & 2.86 & 2.95 & 3.18 & 3.31 \\
\hline $\mathrm{T}_{13} \quad \mathrm{~T}_{2}+$ Castor cake & 1.02 & 1.16 & 1.34 & 1.58 & 1.37 & 1.59 & 1.75 & 2.07 & 2.03 & 2.20 & 2.38 & 2.57 \\
\hline $\mathrm{T}_{14} \quad \mathrm{~T}_{2}+$ Castor cake + Azotobacter & 1.21 & 1.38 & 1.55 & 1.69 & 1.47 & 1.68 & 1.98 & 2.27 & 2.04 & 2.28 & 2.50 & 2.74 \\
\hline $\mathrm{T}_{15} \quad \mathrm{~T}_{2}+$ Castor cake + Arbuscular mycorrhiza & 1.20 & 1.37 & 1.52 & 1.73 & 1.68 & 1.89 & 2.13 & 2.54 & 2.41 & 2.66 & 2.89 & 3.06 \\
\hline $\mathrm{T}_{16} \mathrm{~T}_{3}+$ Neem cake & 1.08 & 1.28 & 1.42 & 1.63 & 1.50 & 1.70 & 1.91 & 2.11 & 2.00 & 2.07 & 2.27 & 2.44 \\
\hline $\mathrm{T}_{17} \mathrm{~T}_{3}+$ Neem cake + Azotobacter & 1.25 & 1.41 & 1.67 & 1.81 & 1.56 & 1.79 & 1.99 & 2.29 & 2.10 & 2.28 & 2.47 & 2.75 \\
\hline $\mathrm{T}_{18} \quad \mathrm{~T}_{3}+$ Neem cake + Arbuscular mycorrhiza & 1.43 & 1.61 & 1.84 & 2.18 & 1.92 & 2.07 & 2.47 & 2.82 & 2.31 & 2.36 & 2.55 & 2.76 \\
\hline $\mathrm{T}_{19} \quad \mathrm{~T}_{3}+$ Castor cake & 1.09 & 1.23 & 1.37 & 1.58 & 1.39 & 1.63 & 1.91 & 2.24 & 1.90 & 2.09 & 2.33 & 2.52 \\
\hline $\mathrm{T}_{20} \quad \mathrm{~T}_{3}+$ Castor cake + Azotobacter & 1.14 & 1.32 & 1.44 & 1.61 & 1.56 & 1.79 & 1.95 & 2.34 & 2.30 & 2.34 & 2.50 & 2.63 \\
\hline $\mathrm{T}_{21} \quad \mathrm{~T}_{3}+$ Castor cake + Arbuscular mycorrhiza & 1.31 & 1.51 & 1.77 & 1.94 & 1.65 & 1.88 & 2.08 & 2.41 & 2.08 & 2.21 & 2.48 & 2.69 \\
\hline Mean & 1.14 & 1.32 & 1.50 & 1.73 & 1.46 & 1.66 & 1.93 & 2.27 & 2.09 & 2.25 & 2.42 & 2.60 \\
\hline $\mathrm{SE}(\mathrm{m}) \pm$ & 0.10 & 0.09 & 0.09 & 0.12 & 0.04 & 0.08 & 0.13 & 0.15 & 0.15 & 0.15 & 0.06 & 0.05 \\
\hline C.D. $(5 \%)$ & 0.30 & 0.27 & 0.27 & 0.36 & 0.12 & 0.24 & 0.39 & 0.44 & 0.43 & 0.43 & 0.18 & 0.14 \\
\hline
\end{tabular}


Table.4 Effect of organic amendments and bio-agents on leaf area $\left(\mathrm{cm}^{2}\right)$ of 2, 3 and 4 months old acid lime seedlings cv. Balaji

\begin{tabular}{|c|c|c|c|c|c|c|c|c|c|c|c|c|}
\hline \multirow{2}{*}{ Treatment } & \multicolumn{4}{|c|}{2 months old } & \multicolumn{4}{|c|}{3 months old } & \multicolumn{4}{|c|}{4 months old } \\
\hline & DAT 60 & DAT 90 & DAT 120 & DAT 150 & DAT 60 & DAT 90 & DAT 120 & DAT 150 & DAT 60 & DAT 90 & DAT 120 & DAT 150 \\
\hline $\mathrm{T}_{1} \quad \mathrm{FYM}+$ Sand +soil (control) & 3.54 & 4.73 & 6.34 & 8.03 & 4.26 & 5.88 & 7.92 & 9.82 & 5.64 & 7.28 & 10.00 & 11.10 \\
\hline $\mathrm{T}_{2} \quad$ Vermicompost + Sand + Soil & 4.48 & 4.97 & 6.62 & 8.38 & 4.97 & 6.93 & 8.60 & 10.72 & 6.96 & 8.49 & 10.91 & 12.27 \\
\hline $\mathrm{T}_{3} \quad$ Cocopeat + Sand + Soil & 4.72 & 5.12 & 6.80 & 8.66 & 5.28 & 7.27 & 9.27 & 11.28 & 5.72 & 7.55 & 9.62 & 11.16 \\
\hline $\mathrm{T}_{4} \quad \mathrm{~T}_{1}+$ Neem cake & 4.41 & 5.64 & 8.25 & 10.45 & 5.21 & 7.88 & 10.77 & 12.94 & 6.70 & 9.03 & 11.74 & 13.21 \\
\hline $\mathrm{T}_{5} \quad \mathrm{~T}_{1}+$ Neem cake + Azotobacter & 4.95 & 6.51 & 8.81 & 10.94 & 5.63 & 7.75 & 10.87 & 12.76 & 6.85 & 8.65 & 12.02 & 13.98 \\
\hline $\mathrm{T}_{6} \quad \mathrm{~T}_{1}+$ Neem cake + Arbuscular mycorrhiza & 5.85 & 7.85 & 10.53 & 12.46 & 6.13 & 7.97 & 10.76 & 12.34 & 7.50 & 9.35 & 12.34 & 14.52 \\
\hline $\mathrm{T}_{7} \quad \mathrm{~T}_{1}+$ Castor cake & 4.67 & 6.71 & 8.34 & 10.35 & 5.78 & 7.18 & 9.57 & 11.96 & 6.04 & 7.63 & 10.21 & 12.56 \\
\hline $\mathrm{T}_{8} \quad \mathrm{~T}_{1}+$ Castor cake + Azotobacter & 5.85 & 7.63 & 9.68 & 11.58 & 6.61 & 8.98 & 11.76 & 13.41 & 7.39 & 9.68 & 12.35 & 13.89 \\
\hline $\mathrm{T}_{9} \quad \mathrm{~T}_{1}+$ Castor cake + Arbuscular mycorrhiza & 5.67 & 7.77 & 9.81 & 11.81 & 6.39 & 9.19 & 11.30 & 13.13 & 7.19 & 9.23 & 11.75 & 14.29 \\
\hline $\mathrm{T}_{10} \quad \mathrm{~T}_{2}+$ Neem cake & 5.13 & 6.61 & 8.11 & 9.68 & 6.14 & 7.83 & 9.45 & 11.93 & 7.21 & 9.03 & 11.25 & 13.37 \\
\hline $\mathrm{T}_{11} \quad \mathrm{~T}_{2}+$ Neem cake + Azotobacter & 6.10 & 8.10 & 10.85 & 13.18 & 6.92 & 9.25 & 11.78 & 14.19 & 6.91 & 8.95 & 11.39 & 12.80 \\
\hline $\mathrm{T}_{12} \quad \mathrm{~T}_{2}+$ Neem cake + Arbuscular mycorrhiza & 6.42 & 8.26 & 10.91 & 13.55 & 7.21 & 9.75 & 13.19 & 16.15 & 7.79 & 10.63 & 13.63 & 16.40 \\
\hline $\mathrm{T}_{13} \quad \mathrm{~T}_{2}+$ Castor cake & 4.97 & 6.65 & 8.76 & 10.99 & 5.92 & 7.85 & 10.45 & 12.53 & 6.93 & 9.38 & 11.86 & 14.08 \\
\hline $\mathrm{T}_{14} \quad \mathrm{~T}_{2}+$ Castor cake + Azotobacter & 5.52 & 7.31 & 9.55 & 11.44 & 5.96 & 8.74 & 11.28 & 13.77 & 7.42 & 10.73 & 13.32 & 14.95 \\
\hline $\mathrm{T}_{15} \mathrm{~T}_{2}+$ Castor cake + Arbuscular mycorrhiza & 6.05 & 8.26 & 10.19 & 12.63 & 6.73 & 8.81 & 11.67 & 14.27 & 8.22 & 11.15 & 13.98 & 16.02 \\
\hline $\mathrm{T}_{16} \mathrm{~T}_{3}+$ Neem cake & 4.95 & 6.16 & 8.57 & 11.22 & 5.32 & 7.34 & 10.77 & 13.55 & 6.32 & 9.42 & 12.30 & 14.15 \\
\hline $\mathrm{T}_{17} \mathrm{~T}_{3}+$ Neem cake + Azotobacter & 5.61 & 7.26 & 9.62 & 12.46 & 5.94 & 7.97 & 10.23 & 12.88 & 7.11 & 9.30 & 12.2 & 13.2 \\
\hline $\mathrm{T}_{18} \quad \mathrm{~T}_{3}+$ Neem cake + Arbuscular mycorrhiza & 6.66 & 8.57 & 11.34 & 14.44 & 7.26 & 9.38 & 12.54 & 15.30 & 7.39 & 9.24 & 13.19 & 14.84 \\
\hline $\mathrm{T}_{19} \quad \mathrm{~T}_{3}+$ Castor cake & 4.98 & 6.95 & 8.89 & 10.77 & 5.96 & 7.98 & 10.78 & 12.79 & 6.7 & 9.84 & 12.62 & 14.35 \\
\hline $\mathrm{T}_{20} \quad \mathrm{~T}_{3}+$ Castor cake + Azotobacter & 5.46 & 7.93 & 10.09 & 11.71 & 6.09 & 8.20 & 11.10 & 13.67 & 7.07 & 9.55 & 12.71 & 15.41 \\
\hline $\mathrm{T}_{21} \quad \mathrm{~T}_{3}+$ Castor cake + Arbuscular mycorrhiza & 5.40 & 6.80 & 8.70 & 11.16 & 6.57 & 8.82 & 12.57 & 14.02 & 7.39 & 9.81 & 13.26 & 15.96 \\
\hline Mean & 5.30 & 6.94 & 9.08 & 11.23 & 6.01 & 8.14 & 10.79 & 13.02 & 6.97 & 9.23 & 12.03 & 13.93 \\
\hline $\mathrm{SE}(\mathrm{m}) \pm$ & 0.26 & 0.38 & 0.43 & 0.56 & 0.35 & 0.27 & 0.43 & 0.52 & 0.66 & 0.20 & 0.25 & 0.46 \\
\hline C.D. (5\%) & 0.75 & 1.10 & 1.23 & 1.62 & 1.01 & 0.78 & 1.25 & 1.50 & N/A & 0.59 & 0.72 & 1.33 \\
\hline
\end{tabular}


Table.5 Effect of organic amendments and bio-agents on SPAD chlorophyll content of 2, 3 and 4 months old acid lime seedlings cv. Balaji

\begin{tabular}{|c|c|c|c|c|c|c|c|c|c|c|c|c|}
\hline \multirow{2}{*}{ Treatment } & \multicolumn{4}{|c|}{2 months old } & \multicolumn{4}{|c|}{3 months old } & \multicolumn{4}{|c|}{4 months old } \\
\hline & DAT 60 & DAT 90 & DAT 120 & DAT 150 & DAT 60 & DAT 90 & DAT 120 & DAT 150 & DAT 60 & DAT 90 & DAT 120 & DAT 150 \\
\hline FYM +Sand +soil (control) & 42.86 & 45.18 & 48.46 & 52.13 & 44.98 & 47.54 & 52.86 & 56.53 & 45.5 & 49.37 & 53.67 & 58.65 \\
\hline $\mathrm{T}_{2} \quad$ Vermicompost + Sand + Soil & 43.19 & 45.86 & 49.23 & 53.18 & 47.41 & 50.55 & 54.62 & 58.23 & 50.35 & 53.33 & 56.74 & 61.60 \\
\hline $\mathrm{T}_{3} \quad$ Cocopeat + Sand + Soil & 43.61 & 46.25 & 50.61 & 54.40 & 45.10 & 48.47 & 53.52 & 57.37 & 48.33 & 51.21 & 55.65 & 59.60 \\
\hline $\mathrm{T}_{4} \quad \mathrm{~T}_{1}+$ Neem cake & 44.36 & 47.59 & 51.24 & 55.29 & 46.28 & 50.29 & 54.84 & 59.17 & 47.27 & 50.16 & 54.29 & 60.45 \\
\hline $\mathrm{T}_{5} \quad \mathrm{~T}_{1}+$ Neem cake + Azotobacter & 46.65 & 48.73 & 52.70 & 57.17 & 49.80 & 52.70 & 56.65 & 60.20 & 52.66 & 57.13 & 60.63 & 64.70 \\
\hline $\mathrm{T}_{6} \quad \mathrm{~T}_{1}+$ Neem cake + Arbuscular mycorrhiza & 49.37 & 52.10 & 55.55 & 58.80 & 51.24 & 54.85 & 59.86 & 63.25 & 54.20 & 58.36 & 62.44 & 69.52 \\
\hline $\mathrm{T}_{7} \quad \mathrm{~T}_{1}+$ Castor cake & 45.17 & 48.62 & 51.16 & 54.10 & 48.06 & 51.84 & 55.77 & 59.50 & 49.37 & 53.26 & 56.33 & 61.73 \\
\hline $\mathrm{T}_{8} \quad \mathrm{~T}_{1}+$ Castor cake + Azotobacter & 46.70 & 48.81 & 51.93 & 56.29 & 49.77 & 52.92 & 57.10 & 61.38 & 51.42 & 55.52 & 59.65 & 64.54 \\
\hline $\mathrm{T}_{9} \quad \mathrm{~T}_{1}+$ Castor cake + Arbuscular mycorrhiza & 47.53 & 50.53 & 53.75 & 57.74 & 51.72 & 54.83 & 59.37 & 63.70 & 52.46 & 56.31 & 59.79 & 65.33 \\
\hline $\mathrm{T}_{10} \quad \mathrm{~T}_{2}+$ Neem cake & 45.83 & 47.14 & 50.71 & 55.08 & 46.96 & 48.75 & 52.77 & 56.37 & 47.44 & 49.91 & 53.53 & 58.44 \\
\hline $\mathrm{T}_{11} \quad \mathrm{~T}_{2}+$ Neem cake + Azotobacter & 47.54 & 51.73 & 54.07 & 58.40 & 49.19 & 52.52 & 56.38 & 61.79 & 49.7 & 53.91 & 56.83 & 61.47 \\
\hline $\mathrm{T}_{12} \quad \mathrm{~T}_{2}+$ Neem cake + Arbuscular mycorrhiza & 51.55 & 53.62 & 57.76 & 62.59 & 53.46 & 56.79 & 61.89 & 67.26 & 55.17 & 59.24 & 62.97 & 69.84 \\
\hline $\mathrm{T}_{13} \quad \mathrm{~T}_{2}+$ Castor cake & 45.15 & 47.88 & 51.31 & 55.32 & 46.92 & 49.83 & 53.50 & 58.64 & 47.43 & 50.84 & 54.73 & 61.30 \\
\hline $\mathrm{T}_{14} \mathrm{~T}_{2}+$ Castor cake + Azotobacter & 48.55 & 51.67 & 54.93 & 59.38 & 52.13 & 55.75 & 59.90 & 64.53 & 53.9 & 56.96 & 61.70 & 67.52 \\
\hline $\mathrm{T}_{15} \quad \mathrm{~T}_{2}+$ Castor cake + Arbuscular mycorrhiza & 49.27 & 53.50 & 56.12 & 60.37 & 51.50 & 54.66 & 58.41 & 62.37 & 53.18 & 55.86 & 61.09 & 66.71 \\
\hline $\mathrm{T}_{16} \mathrm{~T}_{3}+$ Neem cake & 46.37 & 49.74 & 52.25 & 57.29 & 47.61 & 50.79 & 54.63 & 59.85 & 49.31 & 52.43 & 56.35 & 61.25 \\
\hline $\mathrm{T}_{17} \mathrm{~T}_{3}+$ Neem cake + Azotobacter & 47.80 & 50.87 & 54.45 & 59.69 & 48.18 & 51.62 & 55.61 & 60.61 & 50.76 & 54.77 & 59.65 & 66.13 \\
\hline $\mathrm{T}_{18} \quad \mathrm{~T}_{3}+$ Neem cake + Arbuscular mycorrhiza & 52.45 & 55.35 & 58.43 & 63.42 & 52.65 & 55.21 & 59.17 & 65.20 & 54.51 & 57.18 & 62.1 & 68.12 \\
\hline $\mathrm{T}_{19} \quad \mathrm{~T}_{3}+$ Castor cake & 47.85 & 49.79 & 53.16 & 55.45 & 49.98 & 51.37 & 54.68 & 61.18 & 49.70 & 52.43 & 56.81 & 63.58 \\
\hline $\mathrm{T}_{20} \quad \mathrm{~T}_{3}+$ Castor cake + Azotobacter & 49.32 & 52.24 & 54.42 & 57.77 & 51.20 & 53.02 & 57.92 & 63.01 & 51.13 & 53.83 & 60.27 & 65.22 \\
\hline $\mathrm{T}_{21} \quad \mathrm{~T}_{3}+$ Castor cake + Arbuscular mycorrhiza & 50.95 & 52.72 & 56.68 & 58.80 & 52.89 & 55.90 & 59.38 & 65.84 & 52.61 & 56.71 & 61.59 & 67.91 \\
\hline Mean & 47.24 & 50.00 & 53.28 & 57.27 & 49.38 & 52.39 & 56.61 & 61.24 & 50.78 & 54.22 & 58.42 & 63.98 \\
\hline $\mathrm{SE}(\mathrm{m}) \pm$ & 1.25 & 1.14 & 1.32 & 1.48 & 1.50 & 0.80 & 0.87 & 0.97 & 1.88 & 0.42 & 0.27 & 0.32 \\
\hline C.D. $(5 \%)$ & 3.58 & 3.29 & 3.80 & 4.24 & 4.31 & 2.29 & 2.49 & 2.80 & 5.4 & 1.22 & 0.77 & 0.93 \\
\hline
\end{tabular}


Application of vermicompost + Sand + Soil + Neem cake $(20 \mathrm{~g})+$ Arbuscular mycorrhiza (5g) $\mathrm{T}_{12}$ had given significantly maximum stem girth $(2.56 \mathrm{~mm})$ after 150 days transplanting seedlings in 2 months old acid lime seedlings (Table 3). Similarly in 3 months old seedlings maximum stem girth $(3.13 \mathrm{~mm})$ was observed with application of vermicompost + Sand + Soil + Neem cake $(20 \mathrm{~g})+$ Arbuscular mycorrhiza (5g). With regard to the four months old seedlings, highest stem girth have been recorded with $\mathrm{T}_{12}(3.31 \mathrm{~mm})$ followed by $\mathrm{T}_{15}(3.06 \mathrm{~mm})$. Lowest values were recorded in $\mathrm{T}_{1}$ in all aged group seedlings. Similar results were also reported by Sharif et al., (2014) in jujube seedlings, Rajamanickam et al., (2008) and Marcos et al., (2011) in papaya plants.

Application of cocopeat + Sand + Soil + Neem cake $(20 \mathrm{~g})$ + Arbuscular mycorrhiza (5g) $\mathrm{T}_{18}$ had given significantly maximum leaf area $\left(14.44 \mathrm{~cm}^{2}\right)$ per seedlings after 150 days transplanting seedlings in 2 months old acid lime seedlings (Table 4). In case of three $\left(16.15 \mathrm{~cm}^{2}\right)$ and four months $\left(16.40 \mathrm{~cm}^{2}\right)$ old seedlings maximum leaf area was observed in $\mathrm{T}_{12}$ and the all three group aged seedlings lowest leaf area was observed $\mathrm{T}_{1}$ (soli + sand+ FYM). These results are also supported with those of Mohdilyas et al., (2015) in kinnow plants, Patil et al., (2013) in Rangpur lime seedlings and Aseri et al., (2009) in aonla seedlings.

For 2 months old acid lime seedlings maximum SPAD chlorophyll content was recorded in $\mathrm{T}_{18}$ (63.42) (Table 5). In the case of three (67.26) and four (69.84) months old seedlings maximum SPAD chlorophyll content was observed in $\mathrm{T}_{12}$. Lowest values were recorded in $T_{1}$ in all aged group seedlings. Organic amendments helped to release available nutrients to soil such as $\mathrm{N}, \mathrm{P}$, $\mathrm{K}, \mathrm{Mg}$ and $\mathrm{Fe}$ which are required for light harvesting and subsequent conversion in to chemical energy via photo assimilation thus contributed to maximum SPAD chlorophyll content. These results were in conformity with those of Bhagat et al., (2013) in rough lemon, Afsaripoor and Roota (2011) in strawberry plants, Uma and Malathi (2009) in amaranthus plants and Banker et al., (2009) in kagzi lime seedlings.

Increase in plant height, number of leaves, stem girth, leaf area and SPAD chlorophyll content in $\mathrm{T}_{12}$ and $\mathrm{T}_{18}$ which contain organic amendments i.e., vermicompost, cocopeat, neem cake could be due to the fact that these materials besides increasing water holding capacity in the soil also are rich in nutrients (major and micronutrients). Amendments releases nutrient slowly for longer periods, and hence the availability of nutrients to the plants might be continuous. Further the role of AMF in boosting the plant growth and well established in many crops and roots with mycorrhiza have more surface area to absorb water and nutrients and the main role in plant can't directly use the minute amount of phosphorus contained in the soil. Mycorrhiza 'transforms' or solubilize the phosphorus contained in the soil to the benefit of the plant. In the present study also combination of vermicompost, cocopeat, neem cake with AM must have played vital role in enhancing the seedling growth.

In conclusion, the potting mixture containing soil + sand + vermicompost @ 1:1:1 v/v + $A M[5 \mathrm{~g}]$ and neem cake $20 \mathrm{~g}$ per bag was best potting media for both 3 and 4 months old acid lime seedlings grown in the poly bags, whereas in case of 2 months old seedlings best potting mixture was soil + sand + cocopeat@1:1:1 v/v + AM [5g] and neem cake $20 \mathrm{~g}$ per bag.

\section{References}

Anonymous (2015-16) An outline of agriculture situation in Andhra Pradesh 2015-16. Directorate of 
Economics and statistics government of Andhra Pradesh.

Aseri, G, Neelam Jain, K. and Meghwal, P.R. 2009. Influence of biofertilizer on anola establishment and production in Indian Thar desert. Indian Journal of Horticulture. 66 (4):449-455.

Afsharipoor, S. and Roota, H.R. 2011. Effect of different planting beds on growth and development of strawberry in hydroponic and aquaponic systems. Researchgate. 2:61-66.

Banker, S.P, Indi, D.V. and Gud, M.A. 2009. Effect of VAM fungi and Azospirillum on growth and development of Kagzi lime (Citrus aurantifolia L.) seedlings. Journal of Maharashtra Agricultural University. 34(2):183-185.

Bhardwaj, R.L. 2014. Effect of growing media on seed germination and seedling growth of papaya cv.Red lady. African Journal of Science.8 (4):178-184.

Bhagat, S. Anirudh, T. and Dhaliwal, H.S. 2013. Organic amendments influence growth, buddability and budding success in rough lemon (Citrus jambhiri Lush.). Biological Agriculture \& Horticulture. 29 (1):4657.

Ghosh, S.N. and Bersa, K.C. (1997). Proc. National Symp. Citriculture, Nov. 1719, NRC for Citrus, Nagpur, pp. 180 182.

Hazarika, B. N. and Ansari, S. (2007). Biofertilizers in fruit crops. 28-30.

Kamble,S.R,Navale,A.M, and Sonawane, R.B. 2010. Response of Mango Seedlings to VA-Mycorrhizal Inoculation. International Journal of Plant Protection. 2(2):161-164.

Marcos, V. Hernandez, V. Galarza, L.A. Contreras, D.J. Jose, L. Garcia, E. Aguilera, A.M, Castro, E.H. Tovar, J.C. Ortiz, D.T. 2011. Effect of
Glomus mosseae and Entrophospora colombiana on plant growth, production, and fruit quality of 'Maradol' papaya (Carica papaya L.). Scientia Horticultural 128 (2011):255260.

Mohdilyas, Akash Sharma, Sohnikarani, Wali, V.K. and Prashant, B. 2015. Effect of Propagation media and black polybags on growth and survival of budded kinnow. Plants. An International Quarterly Journal of Sciences.10 (2): 579-584.

Ortas, I. Ortakci, D. and Kaya, Z. 2002. Various mycorrhizal fungi propagated on different hosts have different effect on citrus growth and nutrient uptake. Commun. Soil Sci.Plant Anal. 33(1\&2): 259-272.

Patil, R, Waskar, D.P. and Sonkamble, A.M. 2013. Effect of gibberellic acid, urea and neem cake on growth of Rangpur lime (Citrus limonia, Osbeck) seedlings. Theasian Journal of Horticulture. 8(1):285-287.

Qiang-shen Wu, Ying-Ning Zou and Xin-Hua He. 2010. Contribution of Arbuscular mycorrhizal fungus to growth, photosynthesis, root morphology and ionic balance of citrus seedlings under salt stress. Acta Physiology of Plant. 32: 297-304

Rajamanickam, C, Balasubramanyam, S. and Natarajan, S. 2008. Studies on nursery management in papaya (Carica papaya L.). Acta Horticulture. 851:II International Symposium on papaya.

Rakesh, K.Y, Jain, M.C. and Jhakar, R.P. 2012. Effect of media on growth and development of acid lime (Citrus aurantifolia Swingle) seedling with or without Azotobacter. African Journal of Agricultural Research.7 (48):64216426.

Sharif, N., Ishfaq, M., Memon, N. and Riaz, S. 2014. Standardization of potting 
media for nursery raising seedlings of jujube (Zyzyphus mauritiana Lamk.). Journal of Agricultural Technology. 10 (5):1231-1239.

Sharma, S.D., Pramod, K., Singh, S.K. and Patel, V.B. 2009. Indigenous AM Fungi and Azotobacter isolates, and their screening from citrus seedlings of different levels of inorganic fertilizers application. Indian Journal of Horticulture. 66 (2):183-189.

Uma, B. and Malathi, M. 2009. Vermicompost as a soil supplement to improve growth and yield of Amaranthus species. Research Journal of Agriculture and Biological Sciences. 5(6):1054-1060.

\section{How to cite this article:}

Rajesh Naik, S.M., K.T. Venkataramana, L. Mukundalakshmi, R. Nagaraju and Srinivas Rao, G. 2018. Studies on the Effect of Organic Amendments and Bio-agents on Seedling Growth of Acid Lime (Citrus aurantifolia Swingle) cv. Balaji. Int.J.Curr.Microbiol.App.Sci. 7(07): 78-89. doi: https://doi.org/10.20546/ijcmas.2018.707.010 